Research Article

www.jestr.org

\title{
Kinematic Analysis of HALF Parallel Robot
}

\author{
Semaan Amine and Eddie Gazo Hanna \\ Department of Mechanical Engineering, Beirut Arab University, P.O. Box 11-5020, Riad El Solh, 11072809 Beirut, Lebanon
}

Received 1 July 2019; Accepted 20 September 2019

\begin{abstract}
In the present study, the kinematics of a class of parallel manipulators with two translational and one rotational degrees-offreedom are addressed through the analysis of HALF robot. A detailed kinematic and constraint analysis of the robot is conducted. In addition, an exhaustive singularity characterization is presented with interpretation of the robot's behavior in singular poses. The implications of this study will initiate further investigations on the design of parallel manipulators belonging to the class of manipulators under consideration.
\end{abstract}

Keywords: Robot kinematics, Lower mobility, Parallel mechanism, screw theory.

\section{Introduction}

Parallel Manipulators (PMs) with three degrees-of-freedom (DOFs) have been widely investigated in robotics research [1, 7]. The famous Delta robot $[8,9]$ is the first design involving the parallelogram concept. It allows the output link to remain at a fixed orientation with respect to an input link. Delta robot can output three translational degrees-of-freedom. Later on, a three-DOF spatial parallel manipulator with two translational and one rotational DOFs named HALF was proposed [10]. It was first introduced in two versions: one with prismatic actuators and the other with revolute ones. The advantages of this manipulator are the combination of spatial translational and rotational motions, the usage of parallelogram joint in one of its legs, and the high rotational capability of its endeffector. In 2004, a family of parallel manipulators with two translational and one rotational DOFs was introduced [11] based on the concept of the HALF design. HANA robot, one of the aforementioned family, is constructed by using two legs with parallelogram joints. Later on, several works, for instance $[12,13]$, addressed the kinematics and design of PMs belonging to the aforementioned family.

Screw theory [14-18] and Grassmann-Cayley algebra [1922] have been widely implemented in the kinematics and singularity analysis of parallel mechanisms. The main feature of Grassmann-Cayley algebra is the possibility to provide comprehensive geometric and vector forms of parallel mechanisms' singularity conditions. This paper mainly focuses on a detailed kinematics and singularity analysis of PMs with two independent translations and one rotation through the examination of HALF robot. To the best of the authors' knowledge, such an investigation is presented for the first time in this paper. Furthermore, this class of mechanisms has not been analyzed using Grassmann-Cayley algebra and its superbracket decomposition.

The present paper approaches the kinematics and singularities of a family of PMs with two translational and one

*E-mail address: s.amin@bau.edu.lb

ISSN: 1791-2377 @ 2019 School of Science, IHU. All rights reserved.

doi:10.25103/jestr.125.23 rotational DOFs through the geometric analysis of HALF robot. First the tools and techniques used in the study are introduced. A CAD model of HALF is then presented. Furthermore, the paper conducts a detailed kinematics and constraint analysis of the robot and an exhaustive singularity analysis including serial (leg) singularities and parallel singularities [21]. Finally, a full interpretation of the singular configurations is presented with illustrations and an analysis of the robot's behavior in singular poses. The paper concludes with highlights of the obtained results and potential applications based on its findings.

\section{Theoretical background}

\subsection{Screw theory}

Screw theory began with Plücker's research on line geometry in the second half of the 19th century [14-18]. The instantaneous motion of a rigid body and its force/couple may be described as screws which are denoted by twist and wrench, respectively. Thus, screws can be used to describe the constraints that a spatial rigid mechanism is subjected to and its free motion under the constraints. Additionally, a screw is determined by its axis and pitch in geometry, which offers excellent ease for applying and promoting the theory of screws.

Screws are used in the paper in the forms of twists and wrenches, respectively, to represent the motions of the robot's kinematic joints and the constraint forces/torques exerted on its legs and moving platform. A zero-pitch screw can be written as:

$$
\$^{0}=\left(s^{T} ; r_{a} \times s^{T}\right)^{T}
$$

where $s$ is a unit vector along the screw axis and $r_{a}$ is the position vector of a point a on the screw axis. In turn, an infinite-pitch screw is expressed as:

$\$^{\infty}=\left(0^{T} ; s^{T}\right)^{T}$ 
Zero-pitch screws represent rotations and forces while infinite-pitch screws represent translations and torques.

\subsection{Projective space}

In the three-dimensional projective space, there is one plane at infinity [20]. Let lower-case letters represent finite projective points and upper-case letters represent projective points at infinity. A zero-pitch screw corresponds to a finite line in the projective space and can be written as:

$$
\$^{0}=\left(s^{T} ; r_{a} \times s^{T}\right)^{T}=\mathrm{aS}
$$

where $\mathrm{S}$ is the point at infinity in the $s$ direction. All lines parallel to $s$ intersect in the plane at infinity at point $\mathrm{S}$. In turn, an infinite-pitch screw corresponds to a line at infinity in the projective space and can be written as:

$$
\$^{\infty}=\left(0^{T} ; s^{T}\right)^{T}=\mathrm{UV}
$$

where $\mathrm{U}$ and $\mathrm{V}$ are the points at infinity in the $u$ and $v$ directions, respectively. Vectors $u$ and $v$ are two independent vectors orthogonal to $s$.

\subsection{Bracket and super-bracket}

In Grassmann-Cayley algebra [22], the basic elements are extensors with a given step. Extensors of step 1, 2, and 3, represent points, lines, and planes, respectively. A bracket [abcd] corresponds to the determinant of the $4 * 4$ matrix whose columns are the Plücker coordinates of points $a, b, c$, and $\mathrm{d}$. A bracket is null whenever the tetrahedron from by the four points vanishes, namely, when the four points are coplanar, collinear, or coincident. Since all points at infinity belong to one plane (the unique plane at infinity), a bracket with four points at infinity is always null. Similarly, a bracket with repeated points is null.

A super-bracket $\quad\left[\begin{array}{llllll}a b & c d & \text { ef } & \text { gh } & \mathrm{ij} & \mathrm{kl}\end{array}\right]$ corresponds to the determinant of a $6 * 6$ matrix whose columns are Plücker lines corresponding to projective lines, namely, to screws of either zero- or infinite- pitches. In robot kinematics, the superbracket represents the determinant of a certain $6 * 6$ Jacobian matrix whose examination provides conditions for robot singularities.

Using the super-bracket decomposition [19], a superbracket $\left[\begin{array}{llllll}a b & c d & \text { ef } & \text { gh } & \text { ij } & \mathrm{kl}\end{array}\right]$ can be transformed to 24 monomials, each monomial being a product of 3 brackets. Using geometric properties among the super-bracket points and vanishing conditions of a bracket, the 24 monomials can be reduced. Further manipulation of the nonzero monomials in Grassman-Cayley algebra yields algebraic and vector forms of singularity conditions.

\section{Kinematics of HALF robot}

\subsection{HALF robot}

Fig. 1. shows a CAD model of HALF robot. HALF is a parallel manipulator with three legs $L_{1}, L_{2}$, and $L_{3}$. Legs $L_{1}$ and $L_{2}$ have the $P R U$ structure while leg $L_{3}$ is a $P R \Pi R$ leg. $P, R, U$, and $\Pi$ stand for prismatic, revolute, universal, and planar parallelogram [23] joints, respectively.
In each leg, the prismatic joint is actuated. Leg $L_{i}=P_{i} R_{i 1} R_{i 2} R_{i 3}, \quad \mathrm{i}=1,2$, comprises: a prismatic joint allowing translations along $z$, a revolute joint of axis parallel to $x$ and passing through point $\mathrm{a}_{\mathrm{i}}$, a revolute joint of axis parallel to $x$ and passing through point $b_{i}$, and a revolute joint of axis parallel to $y$ and passing through point $b_{i}$. The last two revolute joints thus form a universal joint. It is noteworthy that:

$\mathrm{a}_{\mathrm{i}} \mathrm{b}_{\mathrm{i}} \perp x, \mathrm{i}=1,2$

$\mathrm{b}_{1} \mathrm{~b}_{2} \| y$

Leg $L_{3}=P_{3} R_{31} \Pi_{3} R_{32}$ comprises: a prismatic joint allowing translations along $z$, a revolute joint of axis parallel to $y$ and passing through point $\mathrm{a}_{3}$, a parallelogram joint allowing translations along $\mathrm{a}_{3} \mathrm{~b}_{3} \times n$, and a revolute joint of axis parallel to $y$ and passing through point $b_{3}$. Notice that:

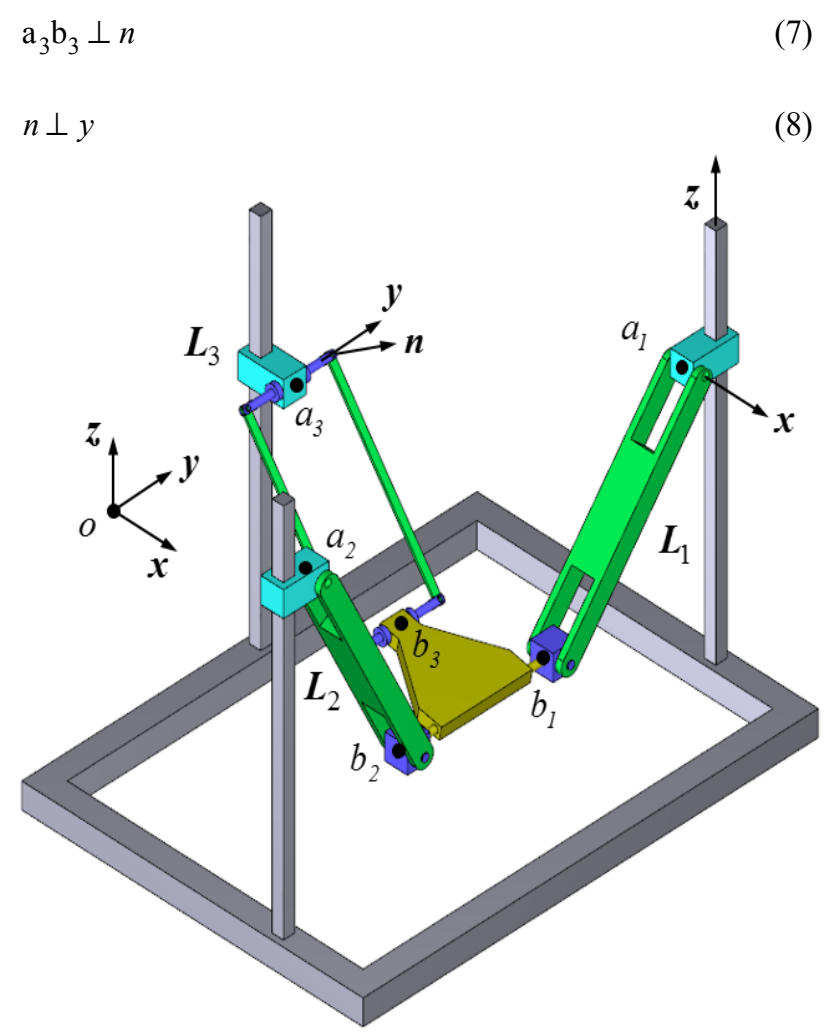

Fig. 1. CAD model of HALF robot.

\subsection{Kinematics of PRU leg}

The twists associated with the kinematic joints of leg $L_{i}$, $\mathrm{i}=1,2$, are:

$$
\begin{aligned}
& T_{i}^{\infty 1}=\left(0^{T} ; z^{T}\right)^{T}=(0,0,0,0,0,1)^{T} \\
& T_{i}^{01}=\left(x^{T} ;\left(r_{a_{i}} \times x\right)^{T}\right)^{T}=\left(1,0,0,0, \mathrm{z}_{\mathrm{a}_{\mathrm{i}}},-\mathrm{y}_{\mathrm{a}_{\mathrm{i}}}\right)^{T} \\
& T_{i}^{02}=\left(x^{T} ;\left(r_{b_{i}} \times x\right)^{T}\right)^{T}=\left(1,0,0,0, \mathrm{z}_{\mathrm{b}_{\mathrm{i}}},-\mathrm{y}_{\mathrm{b}_{\mathrm{i}}}\right)^{T}
\end{aligned}
$$


$T_{i}^{03}=\left(y^{T} ;\left(r_{b_{i}} \times y\right)^{T}\right)^{T}=\left(0,1,0,-\mathrm{z}_{\mathrm{b}_{\mathrm{i}}}, 0, \mathrm{x}_{\mathrm{b}_{\mathrm{i}}}\right)^{T}$ yields:

Applying a linear transformation on the four twist screws $\operatorname{span}\left(T_{i}^{\infty 1}, T_{i}^{01}, T_{i}^{02}, T_{i}^{03}\right)=\operatorname{span}\left(T_{i}^{\infty 1}, T_{i}^{01}, T_{i}^{\infty 2}, T_{i}^{03}\right)$

where $T_{i}^{\infty 2}=\left(0^{T} ;\left(\mathrm{a}_{\mathrm{i}} \mathrm{b}_{\mathrm{i}} \times x\right)^{T}\right)^{T}$

As a result, $\operatorname{leg} L_{i}, \quad \mathrm{i}=1,2$, is a $2 \mathrm{~T} 2 \mathrm{R}$ leg with two independent translations along $z$ and $\mathrm{a}_{\mathrm{i}} \mathrm{b}_{\mathrm{i}} \times x$ in addition to two independent rotations about axes that are parallel to the xoy plane. by:

The kinematic Jacobian matrix of leg $L_{i}, \mathrm{i}=1,2$, is given

$$
J_{P_{i} R_{i} U_{i}}=\left[\begin{array}{cccc}
0 & x & 0 & y \\
z & r_{a_{i}} \times x & \mathrm{a}_{\mathrm{i}} \mathrm{b}_{\mathrm{i}} \times x & r_{b_{i}} \times y
\end{array}\right]
$$

Note that:

$$
\left(\mathrm{a}_{\mathrm{i}} \mathrm{b}_{\mathrm{i}} \times x\right) \times z=\left(0, \mathrm{y}_{\mathrm{a}_{\mathrm{i}} \mathrm{b}_{\mathrm{i}}}, \mathrm{z}_{\mathrm{a}_{\mathrm{i}} \mathrm{b}_{\mathrm{i}}}\right) \times(0,0,1)=\left(0, \mathrm{z}_{\mathrm{a}_{\mathrm{i}} \mathrm{b}_{\mathrm{i}}},-\mathrm{y}_{\mathrm{a}_{\mathrm{i}} \mathrm{b}_{\mathrm{i}}}\right) .
$$

Accordingly, the constrained motions of leg $L_{i}, \mathrm{i}=1,2$, are the rotational DOF about axes that are parallel to $z$ and translations along $x$.

\subsection{Kinematics of PRחR leg}

The twist screws associated with the kinematics joints of leg $L_{3}$ are:

$T_{3}^{\infty 1}=\left(0^{T} ; z^{T}\right)^{T}=(0,0,0,0,0,1)^{T}$

$T_{3}^{01}=\left(y^{T} ;\left(r_{a_{3}} \times y\right)^{T}\right)^{T}=\left(0,1,0,-\mathrm{z}_{\mathrm{a}_{3}}, 0, \mathrm{x}_{\mathrm{a}_{3}}\right)^{T}$

$T_{3}^{\infty 2}=\left(0^{T} ;\left(\mathrm{a}_{3} \mathrm{~b}_{3} \times n\right)^{T}\right)^{T}$

$T_{3}^{02}=\left(y^{T} ;\left(r_{b_{3}} \times y\right)^{T}\right)^{T}=\left(0,1,0,-\mathrm{z}_{\mathrm{b}_{3}}, 0, \mathrm{x}_{\mathrm{b}_{3}}\right)^{T}$

Applying a linear transformation on the four twist screws yields:

$\operatorname{span}\left(T_{3}^{\infty 1}, T_{3}^{01}, T_{3}^{02}, T_{3}^{03}\right)=\operatorname{span}\left(T_{3}^{\infty 1}, T_{3}^{01}, T_{3}^{\infty 2}, T_{3}^{\infty 3}\right)$

where $T_{3}^{\infty 3}=\left(0^{T} ;\left(\mathrm{a}_{3} \mathrm{~b}_{3} \times y\right)^{T}\right)^{T}$

As a result, $L_{3}$ is a $3 \mathrm{~T} 1 \mathrm{R}$ leg with three independent translations along $z, \mathrm{a}_{3} \mathrm{~b}_{3} \times n$, and $\mathrm{a}_{3} \mathrm{~b}_{3} \times y$ in addition to one rotational DOF about an axis that is parallel to $y$.

The kinematic Jacobian matrix of leg $L_{3}$ is given by:

$$
J_{P R \Pi R}=\left[\begin{array}{cccc}
0 & y & 0 & 0 \\
z & r_{a_{3}} \times y & \mathrm{a}_{3} \mathrm{~b}_{3} \times n & \mathrm{a}_{3} \mathrm{~b}_{3} \times y
\end{array}\right]
$$

The constrained motions are rotations about axes that are parallel to $x \mathrm{oz}$ plane.

\section{Constraint analysis of HALF robot}

\subsection{Constraint analysis of PRU leg}

Applying screw reciprocity conditions to the twists screws of leg $L_{i}, \mathrm{i}=1,2$, its constraint wrench screws are a constraint force $W_{i}^{0 c}$ and a constraint couple $W_{i}^{\infty c}$ expressed as follows:

$\left\{\begin{array}{l}W_{i}^{0 c}=\left(x^{T} ;\left(r_{b_{i}} \times x\right)^{T}\right)^{T} \\ W_{i}^{\infty c}=\left(0^{T} ; z^{T}\right)^{T}\end{array}\right\} \quad \mathrm{i}=1,2$

Similarly, the actuation wrench of $\operatorname{leg} L_{i}, \mathrm{i}=1,2$, is a force $W_{i}^{0 a}$ as follows:

$$
W_{i}^{0 a}=\left(\mathrm{a}_{\mathrm{i}} \mathrm{b}_{\mathrm{i}}{ }^{T} ;\left(r_{b_{i}} \times \mathrm{a}_{\mathrm{i}} \mathrm{b}_{\mathrm{i}}\right)^{T}\right)^{T}, \mathrm{i}=1,2
$$

\subsection{Constraint analysis of PRחR leg}

Applying the reciprocity of screws to the twist screws of leg $L_{3}$, its constraint wrench screws are two constraint couples $W_{3}^{\infty c 1}$ and $W_{3}^{\infty c 2}$ expressed as follows:

$W_{3}^{\infty c 1}=\left(0^{T} ; x^{T}\right)^{T}$
$W_{3}^{\infty c 2}=\left(0^{T} ; z^{T}\right)^{T}$

As for the actuation wrench of leg $L_{3}$, it is a force $W_{3}^{0 a}$ as follows:

$$
W_{3}^{0 a}=\left(\mathrm{a}_{3} \mathrm{~b}_{3}^{T} ;\left(r_{b_{3}} \times \mathrm{a}_{3} \mathrm{~b}_{3}\right)^{T}\right)^{T}
$$

\subsection{Platform's constraints}

The moving platform's constraint wrench system spans the constraint wrench systems of legs $L_{1}, L_{2}$, and $L_{3}$ that are expressed in Eqs. (11) and (13). Notice that $W_{1}^{\infty c}=W_{2}^{\infty c}=W_{3}^{\infty c 2}$.

Moreover:

$\operatorname{span}\left(W_{1}^{0 c}, W_{2}^{0 c}\right)=\operatorname{span}\left(W_{1}^{0 c}, W_{12}^{\infty c}\right)=\operatorname{span}\left(W_{2}^{0 c}, W_{12}^{\infty c}\right)$

where

$W_{12}^{\infty c}=\left(0^{T} ;\left(\mathrm{b}_{1} \mathrm{~b}_{2} \times x\right)^{T}\right)^{T}=\left(0^{T} ; z^{T}\right)^{T}$

The constraint wrench system of HALF robot is given by:

$\operatorname{span}\left(W_{1}^{0 c}, W_{1}^{\infty c}, W_{3}^{\infty c 1}\right)$ 
Therefore, the moving platform is constrained to translate along $x$ and to rotate about axes that are parallel to $x 0 z$ plane. The Jacobian of the direct kinematics of HALF robot is represented by:

$$
\begin{aligned}
& J_{d i r}=\left[\begin{array}{cccccc}
W_{1}^{0 c} & W_{1}^{\infty c} & W_{3}^{\infty c 1} & W_{1}^{0 a} & W_{2}^{0 a} & W_{3}^{0 a}
\end{array}\right] \\
& =\left[\begin{array}{cccccc}
x & 0 & 0 & \mathrm{a}_{1} \mathrm{~b}_{1} & \mathrm{a}_{2} \mathrm{~b}_{2} & \mathrm{a}_{3} \mathrm{~b}_{3} \\
r_{b_{i}} \times x & z & x & r_{b_{1}} \times \mathrm{a}_{1} \mathrm{~b}_{1} & r_{b_{2}} \times \mathrm{a}_{2} \mathrm{~b}_{2} & r_{b_{3}} \times \mathrm{a}_{3} \mathrm{~b}_{3}
\end{array}\right]
\end{aligned}
$$

It is noteworthy that $W_{1}^{0 c}$ may be replaced with $W_{2}^{0 c}$.

\section{Singularity analysis}

Awareness of singular poses is of major importance for the design and implementation of robot architectures. A detailed singularity analysis of HALF robot is conducted in this section. Leg singularities are first addressed before investigating the parallel singularities.

Leg singularities relate to the leg's kinematic screw matrix and occur when the leg's kinematic twists become linearly dependent. Such singularities are associated with a loss of degree(s)-of-freedom.

Parallel singularities relate to the rank deficiency of the direct kinematics' Jacobian matrix. They can be associated with either a loss of control of the moving platform or a change in the constrained motions.

\subsection{PRU leg singularities}

Leg $L_{i}, \mathrm{i}=1,2$, exhibits singularity whenever its kinematic Jacobian matrix expressed in Eq. (9) is rank deficient. Since the second and fourth columns of the concerned matrix are zero-pitch screws along two directions that are independent in any leg or robot configurations, the leg singularities correspond to the linear dependency of the first and third columns which are infinite-pitch screws. Accordingly, leg $L_{i}$ is singular whenever:

- $\quad\left(a_{\mathrm{i}} \mathrm{b}_{\mathrm{i}} \times x\right) \| z$. Since $\mathrm{a}_{\mathrm{i}} \mathrm{b}_{\mathrm{i}} \perp x$, the present conditions means that $\mathrm{a}_{\mathrm{i}} \mathrm{b}_{\mathrm{i}} \| y$. This condition is illustrated for leg $L_{1}$ in Fig. 2.

- $\mathrm{a}_{\mathrm{i}} \mathrm{b}_{\mathrm{i}} \| x$. This condition is impossible to occur for the geometry of leg $L_{i}, \mathrm{i}=1,2$.

\subsection{PRחR leg singularities}

Leg $L_{3}$ is in singularity whenever its kinematic Jacobian matrix expressed in Eq. (10) is rank deficient. Accordingly, singularities of leg $L_{3}$ are related to the linear dependency of the three infinite-pitch twists, namely, the first, third, and fourth columns of Eq. (10). The singularity conditions for leg $L_{3}$ are as follows:

- $\quad a_{3} b_{3} \| x o y$, as illustrated in Fig. 3. Special cases of this condition are $\mathrm{a}_{3} \mathrm{~b}_{3} \| x$ and $\mathrm{a}_{3} \mathrm{~b}_{3} \| y$.
- $\mathrm{a}_{3} \mathrm{~b}_{3} \| n$. Impossible since $\mathrm{a}_{3} \mathrm{~b}_{3} \perp n$ in any robot configuration.

- $\quad \mathrm{a}_{3} \mathrm{~b}_{3} \times n, \mathrm{a}_{3} \mathrm{~b}_{3} \times y$, and $z$ are parallel to one plane. For instance, this occurs if $\mathrm{a}_{3} \mathrm{~b}_{3} \times n$ is orthogonal to $y$.

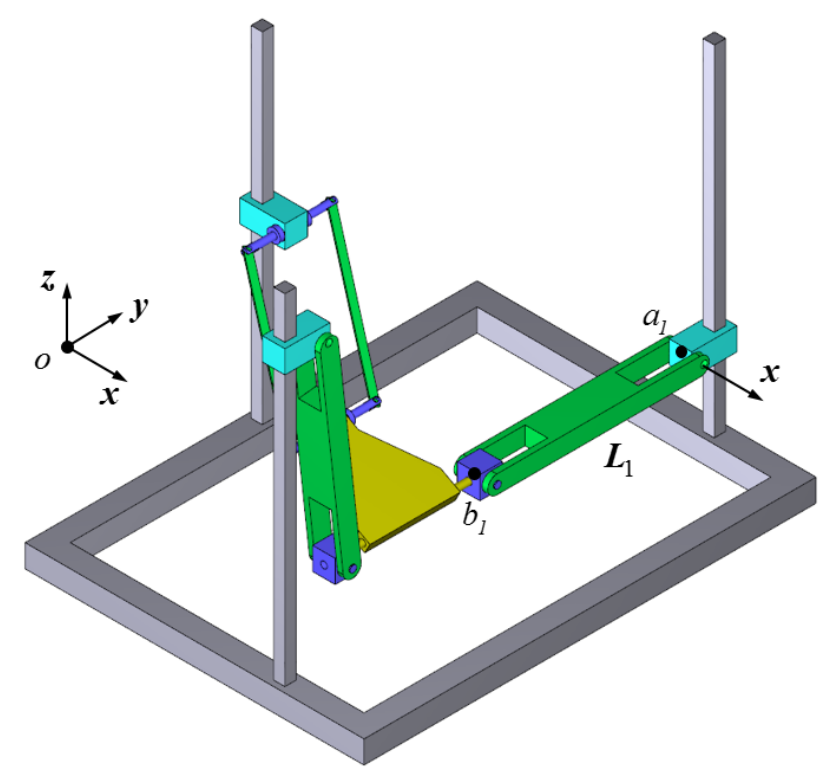

Fig. 2. Singular configuration of leg $L_{1}: \mathrm{a}_{1} \mathrm{~b}_{1} \| y$.

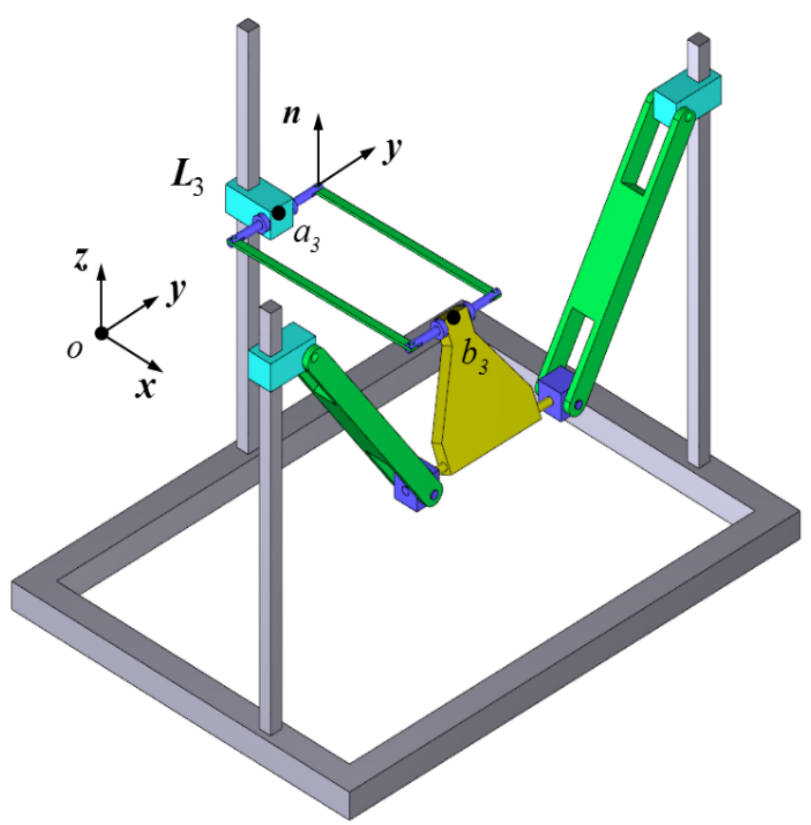

Fig. 3. Singular configuration of leg $L_{3}: \mathrm{a}_{3} \mathrm{~b}_{3} \| x \mathrm{xy}$.

\subsection{Parallel singularities}

A parallel singularity arises whenever the direct Jacobian matrix expressed in Eq. (15) becomes rank deficient. Parallel singularities are examined in this paper for HALF robot using the super-bracket decomposition. To form the required superbracket, it is required to select two points on each of the wrenches (projective lines) constituting the columns of $J_{d i r}$.

In the projective space, let $\mathrm{X}, \mathrm{Y}$, and $\mathrm{Z}$ be the points at infinity in the $x-, y-$, and $z$-directions, respectively. Accordingly, three lines represent the platform's constraint wrench system as follows: 


$$
\begin{aligned}
& W_{1}^{0 c}=\mathrm{b}_{1} \mathrm{X} \\
& W_{1}^{\infty c}=\mathrm{XY} \\
& W_{3}^{\infty c 1}=\mathrm{YZ}
\end{aligned}
$$

In turn, let $\mathrm{F}_{1}, \mathrm{~F}_{2}$, and $\mathrm{F}_{3}$ be the points at infinity in the directions of lines $b_{1} a_{1}, b_{2} a_{2}$, and $b_{3} a_{3}$, respectively. The actuation wrenches are then represented by:

$W_{1}^{0 a}=\mathrm{b}_{1} \mathrm{a}_{1}=\mathrm{b}_{1} \mathrm{~F}_{1}$

$W_{2}^{0 a}=\mathrm{b}_{2} \mathrm{a}_{2}=\mathrm{b}_{2} \mathrm{~F}_{2}$

$W_{3}^{0 a}=\mathrm{b}_{3} \mathrm{a}_{3}=\mathrm{b}_{3} \mathrm{~F}_{3}$

From Eqs. (15), (16), and (17), the super-bracket can be formulated as follows:

$$
\mathrm{S}_{H A L F}=\left[\begin{array}{llllll}
\mathrm{b}_{1} \mathrm{X} & \mathrm{b}_{1} \mathrm{~F}_{1} & \mathrm{~b}_{2} \mathrm{~F}_{2} & \mathrm{~b}_{3} \mathrm{~F}_{3} & \mathrm{XY} & \mathrm{YZ}
\end{array}\right]
$$

Using the geometric properties among the super-bracket points, the 24 monomials of the super-bracket decomposition can be reduced to a certain number of nonzero monomials. For instance, any bracket with repeated points or with four points at infinity is null and will cause the vanishing of the monomial where it appears. Finally, the super-bracket decomposition of Eq. (18) leads to only one nonzero monomial:

$$
\mathrm{S}_{H A L F}=\left[\mathrm{b}_{1} \mathrm{XF}_{1} \mathrm{~F}_{2}\right]\left[\mathrm{b}_{1} \mathrm{~b}_{3} \mathrm{~F}_{3} \mathrm{Y}\right]\left[\mathrm{b}_{1} \mathrm{XYZ}\right]
$$

Parallel singularities occur whenever $\mathrm{S}_{H A L F}$ is null. The singularity conditions turn out to be:

1. $\left[b_{1} X F_{1} F_{2}\right]=0$. This condition requires the collinearity of points $\mathrm{X}, \mathrm{F}_{1}$, and $\mathrm{F}_{2}$ at infinity, which means that vector $x$, line $\mathrm{a}_{1} \mathrm{~b}_{1}$ (whose point and infinity is $\mathrm{F}_{1}$ ), and line $a_{2} b_{2}$ (whose point and infinity is $F_{2}$ ), are parallel to one plane. In vector form, this condition becomes:

$$
\left(\mathrm{a}_{1} \mathrm{~b}_{1} \times \mathrm{a}_{2} \mathrm{~b}_{2}\right) \bullet x=0
$$

Different situations for which Eq. (20) holds are listed below:

i. $\quad a_{1} b_{1} \| a_{2} b_{2}$. Keeping in mind that both lines $a_{1} b_{1}$ and $a_{2} b_{2}$ are orthogonal to $x$ in any robot configuration, the present condition is satisfied in two different situations. First, when $a_{1} b_{1}$ and $\mathrm{a}_{2} \mathrm{~b}_{2}$ are horizontal, namely, parallel to $y$ which means that the two lines coincide. Clearly, this could be avoided by appropriate dimensioning of arms $a_{1} b_{1}$ and $a_{2} b_{2}$. Second, when $a_{1} b_{1}$ and $a_{2} b_{2}$ are parallel but not horizontal which is illustrated in Fig. 4.

ii. $\quad a_{1} b_{1} \| x$. Impossible. iii. $\quad \mathrm{a}_{2} \mathrm{~b}_{2} \| x$. Impossible.

iv. $\mathrm{a}_{1} \mathrm{~b}_{1} \times \mathrm{a}_{2} \mathrm{~b}_{2} \perp x$. Unless $\mathrm{a}_{1} \mathrm{~b}_{1}$ and $\mathrm{a}_{2} \mathrm{~b}_{2}$ are parallel, the present condition appears to be impossible since $a_{1} b_{1} \times a_{2} b_{2}$ is parallel to $x$.

2. $\left[b_{1} b_{3} F_{3} Y\right]=0$. This corresponds to $b_{1} b_{3}, a_{3} b_{3}$ (whose point and infinity is $\mathrm{F}_{3}$ ), and $y$ being parallel to one plane. In vector form. This condition can be written as:

$\left(b_{1} b_{3} \times a_{3} b_{3}\right) \cdot y=0$

Eq. (21) applies in the following cases:

i. $\quad \mathrm{b}_{1} \mathrm{~b}_{3} \| y$. Impossible.

ii. $\quad b_{1} b_{3} \times a_{3} b_{3} \perp y$. This includes situations where $\mathrm{b}_{1} \mathrm{~b}_{3} \| \mathrm{a}_{3} \mathrm{~b}_{3}$ or $\mathrm{a}_{3} \mathrm{~b}_{3} \| y$. It is noteworthy that $\mathrm{a}_{3} \mathrm{~b}_{3} \| y$ corresponds to the flattening of the planar parallelogram joint.

Note that for Eq. (21), $b_{1}$ can be replaced with $b_{2}$ due to the linear transformation performed in Section 4.3 on the two constraint forces applied by legs $L_{1}$ and $L_{2}$.

3. $\left[\mathrm{b}_{1} \mathrm{XYZ}\right]=0 \Rightarrow(x \times y) \bullet z=0$. This condition cannot occur.

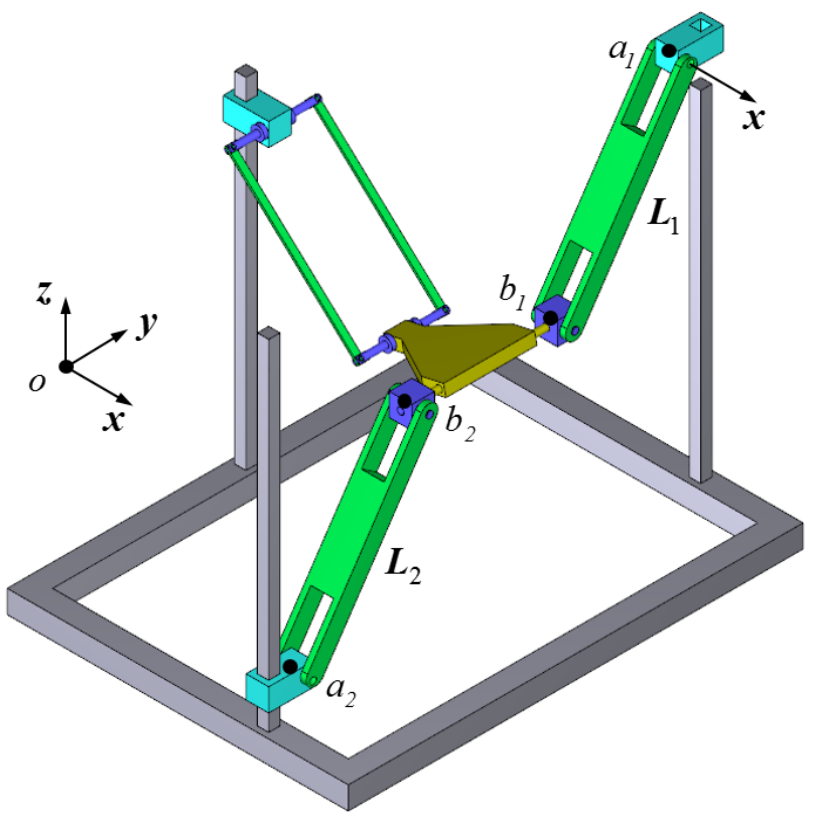

\begin{tabular}{|c|c|c|c|}
\hline \multicolumn{2}{|c|}{$\begin{array}{l}\text { Singularity } \\
\text { condition }\end{array}$} & $\begin{array}{l}\text { Singularit } \\
\text { y type }\end{array}$ & $\begin{array}{c}\text { Associated } \\
\text { behavior }\end{array}$ \\
\hline 1 & $\begin{array}{l}\mathrm{a}_{\mathrm{i}} \mathrm{b}_{\mathrm{i}} \| y, \\
\mathrm{i}=1,2\end{array}$ & $\begin{array}{ll}\text { Leg } & L_{i} \\
\text { singularity } & \end{array}$ & $\begin{array}{l}\text { Leg } L_{i} \\
\text { loses a }\end{array}$ \\
\hline
\end{tabular}

Fig. 4. Parallel singularity of HALF robot: $a_{1} b_{1} \| a_{2} b_{2}$.

\section{Results and Discussion}

Section 5 resulted in 11 singularity conditions of which only 5 conditions appear to be reachable for HALF robot.

Table 1. Possible singularity conditions of HALF robot. 


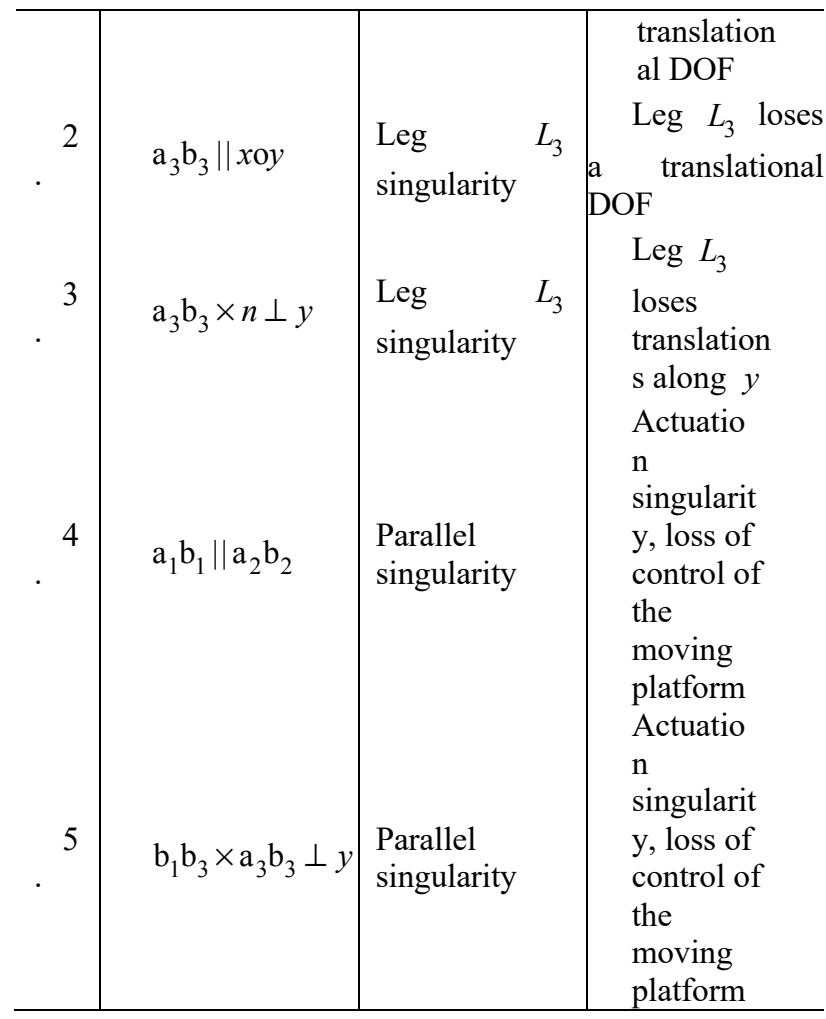

Table 1 recalls the 5 possible singularity conditions. Further discussion of the robot behavior in singular configurations is presented in this section.

1. $\mathrm{a}_{\mathrm{i}} \mathrm{b}_{\mathrm{i}} \| y, \mathrm{i}=1,2$. This is a singularity of leg $L_{i}, \mathrm{i}=1,2$. For instance, for leg $L_{1}$, if $\mathrm{a}_{1} \mathrm{~b}_{1} \| y$, the leg's kinematic Jacobian matrix expressed in Eq. (9) will have two identical columns and thus, the leg loses one translational DOF. In such configurations, the leg provides 1T2R motions, with one possible translational DOF along $z$, rather than its general pattern of 2T2R motions.

2. $\mathrm{a}_{3} \mathrm{~b}_{3} \| x \mathrm{oy}$. This is a singularity of leg $L_{3}$. In particular, if $\mathrm{a}_{3} \mathrm{~b}_{3} \| y$ the leg loses the translational DOF along $\mathrm{a}_{3} \mathrm{~b}_{3} \times y$. Otherwise, if $\mathrm{a}_{3} \mathrm{~b}_{3} \| x \mathrm{x} y$ the leg's Jacobian matrix in Eq. (20) will have two identical columns. Both situations lead leg $L_{3}$ to have $2 \mathrm{~T} 1 \mathrm{R}$ motions rather than its general pattern of $3 \mathrm{~T} 1 \mathrm{R}$ motions.
3. $\mathrm{a}_{3} \mathrm{~b}_{3} \times n \perp y$. In that case the three translational DOFs of leg $L_{3}$ reduce to only two independent translations and thus, the leg loses the translational DOF along $y$.

4. $a_{1} b_{1} \| a_{2} b_{2}$. In that case, the actuation forces applied by legs $L_{1}$ and $L_{2}$ are parallel. Accordingly, the wrenches $W_{1}^{0 a}, W_{2}^{0 a}$, and $W_{1}^{\infty c}$ form a $1-\$^{0}-1-\$^{\infty}$-screw system. In other words, $\operatorname{span}\left(W_{1}^{0 a}, W_{2}^{0 a}, W_{1}^{\infty c}\right)=\operatorname{span}\left(W_{1}^{0 a}, W_{1}^{\infty c}\right)$. Clearly, this is an actuation singularity associated with a loss of control of the moving platform.

5. $\mathrm{b}_{1} \mathrm{~b}_{3} \times \mathrm{a}_{3} \mathrm{~b}_{3} \perp y$. Special cases are $\mathrm{a}_{3} \mathrm{~b}_{3} \| y$ and $b_{1} b_{3} \| a_{3} b_{3}$. Clearly, the constraint wrench system is not affected by this conditions. Therefore, this is also an actuation singularity condition accompanied by a loss of control of the moving platform.

The results clearly show that HALF robot is free of singularities related to the degeneracy of the constraint wrench system, which affect the motion pattern of the moving platform.

\section{Conclusions}

This paper investigated the kinematics and singularities of a family of parallel manipulators with two translational and one rotational DOFs through the geometric analysis of HALF robot. Using the theory of reciprocal screws, a detailed constraint analysis was conducted for each leg and the moving platform. Leg singularities were determined and analyzed. Using the super-bracket decomposition, a detailed parallel singularity analysis was addressed.

To conclude, the paper presented tools to completely list and interpret singular configurations of parallel manipulators with two translational and one rotational DOFs. The obtained results provide better understanding of the geometric properties of this family of manipulators. Moreover, the results would be useful for future works on the type synthesis, singularity avoidance, and design of new parallel manipulators pertaining to the mentioned family.

This is an Open Access article distributed under the terms of the Creative Commons Attribution License

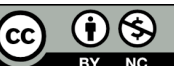

\section{References}

1. K.M. Lee and D.K. Shah, "Kinematic analysis of a three-degrees-offreedom in-parallel actuated manipulator" IEEE Journal on Robotics and Automation, 4 (3), p. 354-360 (1988).

2. C. Gosselin and J. Angeles, "The optimum kinematic design of a spherical three-degree-of-freedom parallel manipulator" Journal of Mechanisms, Transmissions, and Automation in Design, 111 (2), p. 202-207 (1989).

3. L.W. Tsai, "Kinematics of a three-dof platform with three extensible limbs, in: J. Lenarcic, V. Parenti-Castelli (Eds.)" Recent Advances in Robot Kinematics, Springer Netherlands, Dordrecht, p. 401-410 (1996).

4. I. A. Bonev, D. Zlatanov, and C.M.Gosselin, "Advantages of the modified euler angles in the design and control of PKMs", in: 2002 Parallel Kinematic Machines International Conference, p. 171-188 (2002).
5. L. W. Tsai and S. Joshi, "Kinematics and optimization of a spatial 3UPU parallel manipulator”, J. Mechanical Design, 122, p. 439-446 (2000).

6. J. Schadlbauer, D. R. Walter, and M. L. Husty, "A complete kinematic analysis of the 3-RPS parallel manipulator", in: 15th National Conference on Machines and Mechanisms (NaCoMM 2011), India, p. 1-10, (2011).

7. M. Carricato and V. Parenti-Castelli, "Position Analysis of a New Family of 3-DOF Translational Parallel Manipulators", Journal of Mechanical Design, 125 (2), p.1-7, (2003).

8. R. Clavel, "DELTA: a fast robot with parallel geometry", Proc of the 18th International Symposium on Industrial Robots, SpringerVerlag, New York, USA, p.91-100, (1988).

9. X. J. Liu, J. Wang, and H. Zheng, "Workspace atlases for the computer-aided design of the Delta robot", Proceedings of the I 
MECH E Part C: Journal of Mechanical Engineering Science, 217 (8), p. 861-869, (2003)

10. X. J. Liu, J. Wang, and F. Gao, "On the analysis of a new spatial three degrees of freedom parallel manipulator", IEEE Transactions on Robotics and Automation, 17 (6), p.959-968, (2001).

11. X. J. Liu, J. Wang, and G. Pritschow, "A new family of spatial 3DoF fully-parallel manipulators with high rotational capability", Mechanism and Machine Theory, 40 (4), p. 475-494, (2005).

12. X. J. Liu, J. Wang, C. Wu, and J. Kim, "A new family of spatial 3 DOF parallel manipulators with two translational and one rotational DOFs", Robotica, 27, p. 241-247, (2008)

13. Y. Zhang and K. I. Ting, "Design and analysis of a spatial 3-DOF parallel manipulator with 2T1R-type", International Journal of Advanced Robotic Systems, 10, p. 1-8, (2013).

14. R.S. Ball, "A treatise on the theory of screws", Cambridge University Press, Cambridge, (1900).

15. K. H. Hunt, "Kinematic geometry of mechanisms", Oxford Clarendon Press, Oxford, (1978).

16. X. Kong and C. Gosselin, "Type synthesis of parallel mechanisms, Springer, Heidelberg, 33, (2007).

\section{Nomenclature}

$\begin{array}{cl}\text { DOF } & \text { Degree of Freedom } \\ J & \text { Jacobian matrix } \\ \mathrm{p} & \text { Finite point } \\ \mathrm{P} & \text { Point at infinity } \\ P & \text { Prismatic joint } \\ \mathrm{PM} & \text { Parallel Manipulator } \\ R & \text { Revolute joint } \\ \mathrm{S} & \text { Super-bracket } \\ \$ & \text { Screw } \\ T & \text { Twist } \\ U & \text { Universal joint } \\ W & \text { Wrench } \\ \Pi & \text { Planar parallelogram joint }\end{array}$

17. J. Phillips, "Freedom in machinery, introducing screw theory", Cambridge University Press, Cambridge, 1, (1984).

18. J. Phillips, "Freedom in machinery, screw theory exemplified", Cambridge University Press, Cambridge, 2, (1990).

19. P. Ben-Horin and M. Shoham, "Singularity condition of six-degreeof-freedom three-legged parallel robots based on Grassmann-Cayley algebra", IEEE Transactions on Robotics, 22 (4), p. 577-590, (2006).

20. D. Kanaan, P. Wenger, S. Caro, and D. Chablat, "Singularity analysis of lower-mobility parallel manipulators using Grassmann-Cayley algebra", IEEE Transactions on Robotics, 25 (5), p. 995-1004, (2009).

21. S. Amine, M. Tale Masouleh, S. Caro, P. Wenger, and C. Gosselin, "Singularity conditions of 3T1R parallel manipulators with identical limb structures", ASME Journal of Mechanisms and Robotics, 4 (1), p. 1-11, (2012).

22. T. McMillan, "Invariants of antisymmetric tensors", PhD thesis, University of Florida, Gainesville, Florida, USA, (1990).

23. S.Caro, W. A. Khan, D. Pasini, and J. Angeles, "The rule-based conceptual design of the architecture of serial Schonflies-motion generators", Mechanism and Machine Theory, 45 (2), p. 251-260, (2010). 